\title{
The SseC translocon component in Salmonella enterica serovar Typhimurium is chaperoned by SscA
}

Colin A Cooper ${ }^{1,2,3}$, David T Mulder ${ }^{1,2}$, Sarah E Allison ${ }^{1,2}$, Ana Victoria C Pilar ${ }^{1,2}$ and Brian K Coombes ${ }^{1,2^{*}}$

\begin{abstract}
Background: Salmonella enterica is a causative agent of foodborne gastroenteritis and the systemic disease known as typhoid fever. This bacterium uses two type three secretion systems (T3SSs) to translocate protein effectors into host cells to manipulate cellular function. Salmonella pathogenicity island (SPI)-2 encodes a T3SS required for intracellular survival of the pathogen. Genes in SPI-2 include apparatus components, secreted effectors and chaperones that bind to secreted cargo to coordinate their release from the bacterial cell. Although the effector repertoire secreted by the SPI-2 T3SS is large, only three virulence-associated chaperones have been characterized.
\end{abstract}

Results: Here we report that SscA is the chaperone for the SseC translocon component. We show that SscA and SseC interact in bacterial cells and that deletion of sscA results in a loss of SseC secretion, which compromises intracellular replication and leads to a loss of competitive fitness in mice.

Conclusions: This work completes the characterization of the chaperone complement within SPI-2 and identifies SscA as the chaperone for the SseC translocon.

Keywords: Salmonella, Pathogenesis, Chaperone, Translocon, T3SS

\section{Background}

Bacterial pathogens exploit host niches using strategies that block or modify host defense pathways. One such strategy employed by the Gram-negative bacterium Salmonella enterica, is the translocation of effector proteins into the host cell through a type three secretion system (T3SS). S. enterica serovar Typhimurium (S. Typhimurium) has two T3SSs encoded within Salmonella pathogenicity island-1 (SPI-1) and SPI-2 that facilitate invasion and intracellular survival within host cells [1-3]. The assembly of the T3SS is complex, involving the formation of membrane channels in the bacterial inner and outer membrane, and a terminal translocon that forms a pore in host membranes.

Both SPI-1 and SPI-2 encode a distinct group of chaperones that bind to their cognate cargo proteins to

\footnotetext{
*Correspondence: coombes@mcmaster.ca

${ }^{1}$ Michael G. DeGroote Institute for Infectious Disease Research, Hamilton, Ontario L8N 3Z5, Canada

${ }^{2}$ Department of Biochemistry and Biomedical Sciences, McMaster University, Health Sciences Centre Room 4H17, Hamilton, Ontario L8S 4K1, Canada Full list of author information is available at the end of the article
}

coordinate T3SS assembly and secretion of effectors. Virulence chaperones belong to one of three defined classes [4]: class I chaperones bind to single (IA) or multiple (IB) effectors, class II chaperones interact with translocon components, and class III chaperones partner with apparatus components. Among each of the different classes, chaperones share structural similarity yet their amino acid sequence can be poorly conserved. As such, many chaperones have been first identified based on low sequence identity with previously characterized proteins, and by shared physical properties such as isoelectric point (pI). Class I chaperones tend to be small proteins $(\sim 9-15 \mathrm{kDa})$ with acidic pI, and function as dimers adopting a horseshoe-like shape [5-7]. Class II chaperones also form dimers but do not have an acidic pI, which reflects a different interaction surface required for substrate binding $[8,9]$.

In addition to directing secretion events, chaperonecargo pairs can function as regulatory proteins for T3SS gene expression [10]. The FlgN chaperone interacts with FlgK-FlgL to form a repressive complex that inhibits expression of late flagellar genes [11]. The Yersinia

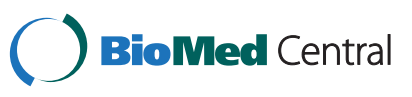


virulence plasmid encodes the chaperone SycD (also known as LcrH) that aids in the secretion of the translocon components YopB and YopD, the latter of which is responsible for establishing a negative feedback loop to prevent effector gene expression at early stages of infection [12]. As SycD is required for YopD stability in the cytosol, both chaperone and cargo are necessary for proper coordination of Yop expression.

In S. enterica, over twenty effectors secreted by the SPI-2 T3SS have been identified yet the full complement of virulence chaperones involved in their secretion remains to be identified or functionally analyzed. To date, three virulence chaperones have been characterized; we showed that SrcA chaperones the effectors SseL and PipB2 and binds to the T3SS ATPase SsaN [5]. The SscB chaperone directs the secretion of SseF [13], and the class II chaperone, SseA, is responsible for the secretion of the putative translocon platform protein $\mathrm{SseB}$ and one of the two translocon proteins, SseD, but not SseC [14-16]. Comparative sequence analysis of SPI-2 identified a putative chaperone gene called $\operatorname{ssc} A$ [17] but its function had yet to be demonstrated. In light of these findings, we set out to identify and characterize the chaperone involved in secretion of the $\mathrm{SseC}$ translocon protein, with an a priori focus on the SscA gene in SPI-2. In this study we demonstrate that SscA interacts with $\mathrm{SseC}$ and is required for its secretion but is dispensable for secretion of the other translocon components SseD and SseB. Both SscA and SseC were required for fitness in infected mice and in vitro macrophage infection assays.

\section{Results}

\section{Identification of SscA as a chaperone for SseC}

SscA was previously predicted to be a chaperone based on comparisons to other T3SS-associated chaperones and therefore we prioritized it for analysis [17]. SscA is an $\sim 18 \mathrm{kDa}$ protein that has $46 \%$ sequence identity to $\mathrm{SycD}$, a translocon chaperone in Yersinia. Using the SycD crystal structure as a model (PDB-2VGY), the secondary structure prediction for SscA [18] showed a solely $\alpha$-helical protein consisting of eight $\alpha$-helices and a large tetratricopeptide repeat (TPR) domain from amino acids 36 to 137 (Figure 1). This helical structure is similar to that found in SycD [8] while the TPR domain has been shown in mutational studies and structural work to be involved in cargo binding for class II chaperones $[19,20]$. Based on this structural comparison, we aimed to further characterize SscA as a potential class II chaperone in the SPI-2 T3SS.

\section{SscA interacts with the translocon protein SseC}

Chaperones exert their biological function in T3SS export through a physical interaction with cargo proteins. Because the class II chaperone SseA binds the translocon components $\mathrm{SseB}$ and $\mathrm{SseD}$ but not $\mathrm{SseC}$, this suggested that another chaperone interacts with $\mathrm{SseC}$. The structural similarity of SscA to SycD made this protein a logical candidate. To test this hypothesis we immunoprecipitated SscA-FLAG from bacterial cells and analyzed the co-precipitated proteins by Western blot with anti-SseC antiserum. SseC was pulled down only in the Salmonella strain expressing SscA-FLAG and not from control lysates generated from untagged wild type cells (Figure 2A). To verify this interaction, we performed a reciprocal co-IP by pulling down SseC-FLAG and showing co-precipitation of SscA-His 6 in the eluted protein fraction (Figure 2B). To examine the specificity of the SscA-SseC interaction, we tested whether SscAFLAG could immunoprecipitate other members of the translocon apparatus, including SseB and SseD, which it did not (Figure 2C). These data indicated that SscA interacted with $\mathrm{SseC}$, but not the other translocon proteins.

\section{SscA is necessary for secretion of SseC}

To determine if the interaction between SscA and SseC was necessary for SseC secretion, we performed an in vitro secretion assay using wild type and $\triangle s s c A$ under conditions that activate expression and activity of the SPI-2 T3SS. The secreted protein fraction from the culture supernatant of both wild type $S$. Typhimurium and $\triangle s s c A$ was immunoblotted for the translocon proteins $\mathrm{SseB}, \mathrm{SseC}$, and SseD using specific antisera. The $s s c A$

$$
\begin{aligned}
& \text { SSCA -MKKDPTLQQAHDTMRFFRRGGLRMLLDDDVTQPLNTLYRYATQLMEVKEFAGAARLFQ } 59 \\
& \text { SyCD MQQETTDTQEYQLAMESFLKGGGTIAMLNE ISSDTLEQLYSLAFNQYQSGKYEDAHKVFQ } 60
\end{aligned}
$$

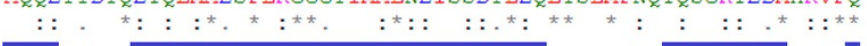

$$
\begin{aligned}
& \text { SSCA LLTIYDAWSFDYWFRLGECCQAQKHWGEAIYAYGRAAQIKIDAPQAPWAAAECYLACDNV } 119 \\
& \text { SyCD ALCVLDHYDSRFFLGLGACRQAMGQYDLAIHSYSYGAIMDIKEPREPFHAAECLLQKGEL } 120
\end{aligned}
$$

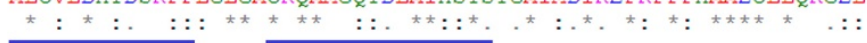

$$
\begin{aligned}
& \text { SSCA CYAIKALKAVVRICGEVSEHQILRQRAEKMLQQLSDRS---------- } 157 \\
& \text { SycD AEAESGLFLAQELIADKPEFKELSTRVSSMLEAIKLKKEMEHEFVDNP } 168
\end{aligned}
$$

Figure 1 Amino acid sequence alignment of SscA and the Yersinia chaperone SycD. Conserved alpha helical regions are denoted with blue bars. Alignment was performed with Clustal W software (www.ebi.ac.uk), alpha helix content was inferred from the published SycD crystal structure (PDB 2VGY) and from predictions made using SSpro8 [21]. 

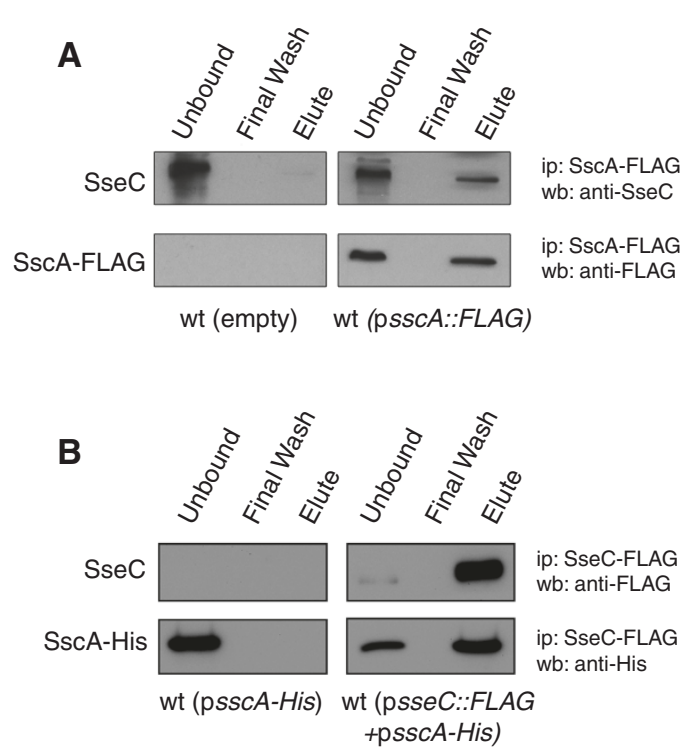

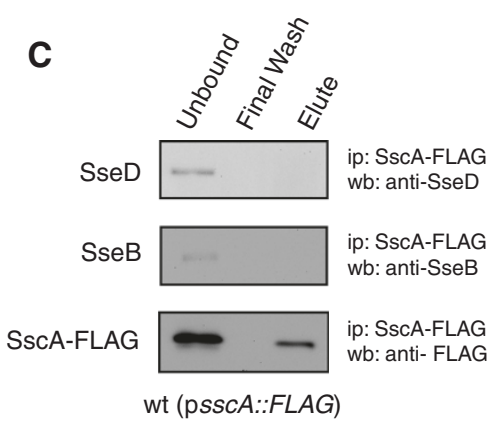

wt (psscA::FLAG)

Figure 2 SscA interacts with the translocon protein SseC. (A) Wild type Salmonella (left panels) and a strain carrying a plasmid expressing SsCA-FLAG (right panels) were grown in LPM minimal medium, lysed and subjected to immunoprecipitation with anti-FLAG antibody. Immunoprecipated proteins were probed by Western blot with anti-SseC antiserum and anti-FLAG antibody. (B) A reciprocal immunoprecipitation to that shown in part A was performed with a strain expressing SscA-His 6 and a strain expressing both SscA-His 6 and SseC-FLAG as indicated. SseC-FLAG was immunoprecipitated and proteins were blotted using anti-His and anti-FLAG antibodies. (C) SsCA-FLAG does not immunoprecipitate the SseB or SseD translocon proteins. The specificity of the SscA-SseC interaction was tested by probing SscA-FLAG immunoprecipitates with antibodies raised against SseD and SseB, neither of which was detectable in the final eluted protein fraction. Each immunoprecipitation experiment was repeated three times with similar results.

mutant failed to secrete $\mathrm{SseC}$ as this protein was absent from the secreted protein fraction despite abundant levels in the bacterial cytoplasmic fraction (Figure 3A). $\mathrm{SseC}$ was detected in both the secreted protein and cytoplasmic fractions from wild type Salmonella and deletion of $s s c A$ had no demonstrable effect on the secretion of SseB or SseD (Figure 3A). To verify this phenotype, we complemented the $\triangle s s c A$ mutant by transforming it with a plasmid to restore $s s c A$ expression. This lead to detectable levels of $\mathrm{SseC}$ in the secreted protein fraction, albeit to lower levels than wild type (Figure 3B), likely due to the increased copy number of SscA relative to its SseC cargo.

\section{SseC and SscA are required for fitness during infection}

Given that SscA was required for secretion of the SseC translocon component, we measured the impact on bacterial fitness following the deletion of $s s e C$ and $s s c A$. Deletion of either $s s c A$ or $s s e C$ reduced the ability of bacteria to survive in RAW264.7 macrophages compared to wild type (Figure 4A). The number of intracellular bacteria between $2 \mathrm{~h}$ and $20 \mathrm{~h}$ after infection was decreased to $10 \%$ of wild type in the $s s e C$ mutant, and to $50 \%$ of wild type in the sscA mutant. To determine whether similar phenotypes could be observed in animal infections, mice were orally gavaged with a mixed inoculum containing equal proportions of wild type and mutant bacteria and the competitive fitness was determined 3 days after infection in the spleen, liver and cecum. The competitive indices for both $s s e C$ and $s s c A$ mutant strains was below 0.20 and were statistically significant (Figure $4 \mathrm{~B}$ and $4 \mathrm{C}$ ). The $\mathrm{CI}$ for the $s s c A$ mutant was 0.18 (95\% CI 0.08-0.27; spleen), 0.19 (95\% CI 0.31-0.35; liver), and 0.13 (95\% CI -0.01-0.20; cecum). Values for the sseC mutant were 0.15 (95\% CI 0.09 0.21 ; spleen), 0.09 (95\% CI 0.04-0.13; liver), and 0.10 (95\% CI -0.01-0.20; cecum). These results indicated that both SseC and SscA are critical for infection of macrophages and for competitive fitness in animals.

\section{Discussion}

Protein-chaperone interactions are essential for T3SS function because they coordinate the delivery and secretion of substrate cargo. Class II virulence chaperones are particularly important since they direct translocon secretion as a prerequisite step for the proper delivery of all subsequent effectors into the host cell. Given the modest sequence similarity between the Yersinia class II virulence chaperone SycD and SscA, we analyzed SscA as the potential chaperone for the $\mathrm{SseC}$ translocon in the Salmonella SPI-2 T3SS. The structure of SycD shows a crescent shape molecule with the concave face possessing protein interaction sites that are common between SycD and SscA (i.e. Y40, Y52, Y93) [8]. The Shigella 


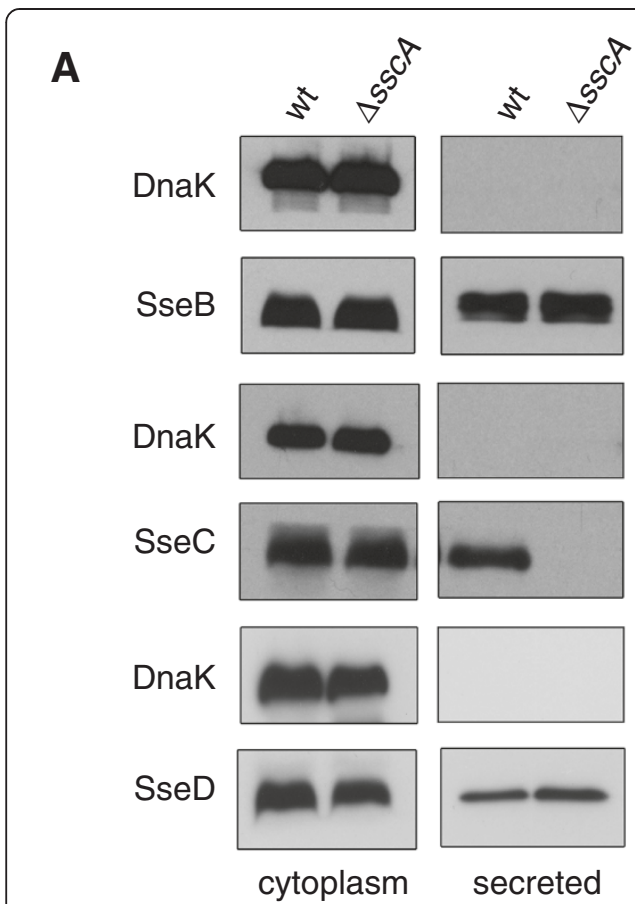

B

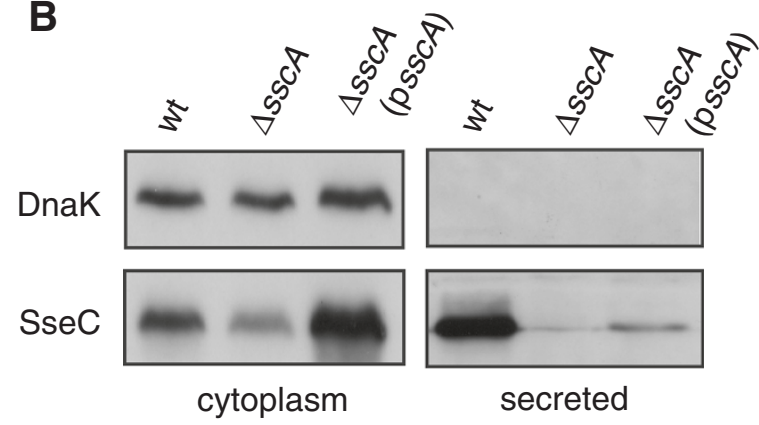

Figure $3 \mathrm{SscA}$ is required for the secretion of SseC. (A) Proteins isolated from the cytoplasm and those secreted into the culture medium by wt and an $\triangle s s c A$ mutant were probed by Western blot for the translocon components SseB, SseC and SseD. All proteins were detected in the cytoplasmic fraction from both strains. Wild type cells secreted each of the translocator apparatus proteins, however, SseC was undetectable in the secreted fraction from $\triangle s s c A$ with no affect on SseB or SseD. Anti-DnaK antibody was used as a control to verify the absence of cytoplasmic protein in the secreted protein fractions. (B) Complementation of $\triangle s s c A$ modestly restores $\mathrm{SseC}$ secretion. Whole cell lysates and secreted protein fractions from wild type, $\triangle s s c A$, and $\triangle s s c A$ transformed with a plasmid encoding SSCA were probed for SseC by Western blot. SseC was detected in the secreted fraction from complemented $\triangle s s c A$, albeit to lower levels than that seen from wild type cells. Secretion experiments were performed three times with similar results.

class II chaperone IpgC possesses a similar structure with the concave face binding an amino acid region of its cognate cargo IpaD [22], suggesting that a common cargo-binding region may exist among class II virulence chaperones.

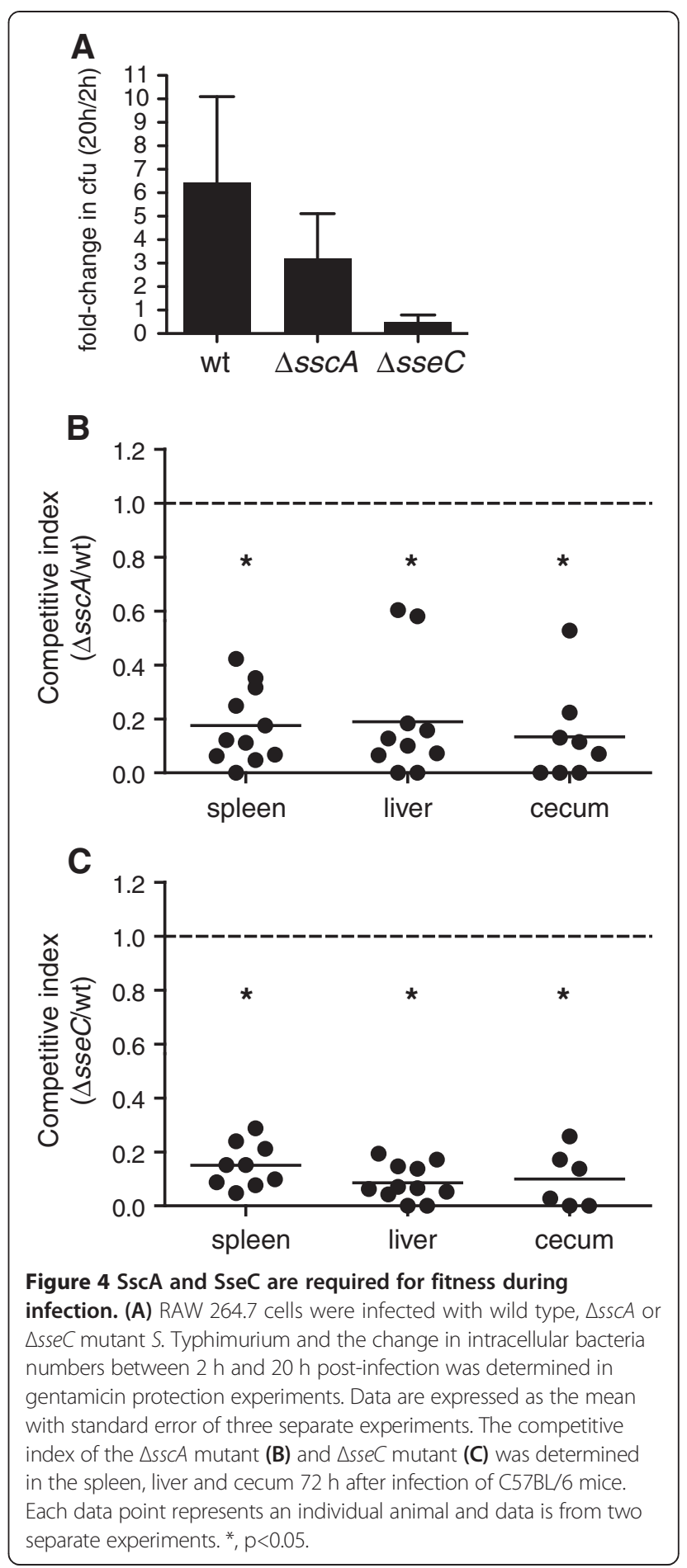

Using protein-protein interaction studies and secretion assays we demonstrated that SscA is the class II virulence chaperone for $\mathrm{SseC}$ and showed that this interaction is important for Salmonella pathogenicity as deletion of either sscA or sseC lead to similar attenuated phenotypes in mouse infections. As documented previously, effectors can be secreted to the cell surface of the 
bacteria in the absence of a functional translocon, however delivery of effector proteins into host cells requires an assembled translocon apparatus [23,24]. Interestingly, the $s s e C$ mutant had a more pronounced negative effect on replication in RAW264.7 cells suggesting an additional role for $\mathrm{SseC}$ that does not depend on its secretion, or that a very small number of bacteria assemble a functional translocon in the absence of the SscA chaperone, allowing for some measure of phenotype recovery in vitro. This latter possibility was suggested for Yersinia LcrH point mutants that had reduced secretion of translocon proteins but retained some ability to intoxicate host cells from a minimal number of T3SS [25]. In our system, we find this possibility unlikely because we found no evidence for $\mathrm{SseC}$ secretion in the absence of SscA chaperone even for highly concentrated samples, and the attenuation level of the $s s c A$ and $s s e C$ mutants was similar in animal infections.

\section{Methods}

\section{Ethics statement}

All experiments with animals were conducted according to guidelines set by the Canadian Council on Animal Care. The local animal ethics committee, the Animal Review Ethics Board at McMaster University, approved all protocols developed for this work.

\section{Bacterial strains, cloning, and growth conditions}

Salmonella enterica serovar Typhimurium strain SL1344 (S. Typhimurium) was used as the wild type parent strain for all mutants generated in this study. Genomic mutations were made using the lambda red mutagenesis method [26]. Electrocompetent cells containing the helper plasmid pKD46 were transformed with PCRamplified linear DNA cassettes encoding the kanamycin resistance gene from the template plasmid pKD4 and flanked with 50 bp ends homologous for the targeted gene. Successful recombinants were recovered by antibiotic selection and screened by PCR for correct gene disruption. To generate FLAG-epitope fusion proteins for immunoprecipitation experiments, the full-length ssc $A$ gene was cloned into the plasmid pFLAG-CTC (Sigma) as a HindIII/SalI fragment and full-length $s s e C$ was cloned as an NdeI/Sall fragment using the primers listed in Table 1. Expression was induced from $\mathrm{P}_{t a c}$ using IPTG. To generate an $s s c A$ fusion to the hexa-histidine $\left(\mathrm{His}_{6}\right)$ epitope, full-length $s s c A$ was amplified from chromosomal SL1344 DNA using primers that incorporated the $\mathrm{His}_{6}$ epitope (Table 1). This fragment was cloned first into pBAD24 [27] as an NheI/HindIII fragment and then subcloned along with $\mathrm{P}_{\mathrm{BAD}}$ into pACYC (New England Biolabs) as a BamHI/HindIII fragment to generate an arabinose-inducible $\mathrm{sscA}-\mathrm{Hi}_{6}$ fusion. Strains, plasmids and primer sequences used in this work are provided in Table 1. The minimal medium LPM at $\mathrm{pH} 5.8$ $\left(5 \mathrm{mM} \mathrm{KCl}, 7.5 \mathrm{mM}\left(\mathrm{NH}_{4}\right)_{2} \mathrm{SO}_{4}, 0.5 \mathrm{mM} \mathrm{K}_{2} \mathrm{SO}_{4}, 80 \mathrm{mM}\right.$ MES, $38 \mathrm{mM}$ glycerol, $0.1 \%$ casamino acids, $8 \mu \mathrm{M} \mathrm{MgCl}_{2}$, $337 \mu \mathrm{M} \mathrm{PO}_{4}^{3-}$ ), was used to induce the expression of SsrBregulated genes as described previously [28]. Bacterial cultures were grown at $37^{\circ} \mathrm{C}$ with shaking at $225 \mathrm{rpm}$ unless otherwise indicated.

\section{Immunoprecipitation experiments}

Co-immunoprecipitations (co-IP) were performed with $S$. Typhimurium expressing SscA-FLAG or SseC-FLAG from the IPTG-inducible pFLAG-CTC plasmid. A strain carrying empty plasmid was used as a control. Strains were grown overnight in Luria-Bertani broth (LB) and sub-cultured 1:50 into $50 \mathrm{ml}$ of LPM medium and grown to an optical density $\left(\mathrm{OD}_{600}\right)$ of 0.6 at $37^{\circ} \mathrm{C}$. Cultures were then centrifuged at $3000 \times g$ for $10 \mathrm{~min}$, and resuspended in phosphate buffered saline (PBS) containing mini-EDTA protease inhibitor cocktail (PBS-PI; Roche). Cells were lysed by 6 pulses of sonication for $30 \mathrm{sec}$ each, with $60 \mathrm{sec}$ intervals between sonication (Misonix Sonicator 3000, Misonix). Lysates were centrifuged at $3000 \times g$ for 15 min at $4{ }^{\circ} \mathrm{C}$ and the supernatant removed to obtain the cytosolic protein fraction. M2-agarose beads conjugated with anti-FLAG antibodies (F-gel; Sigma) was equilibrated with PBS-PI containing $10 \mu \mathrm{g} / \mathrm{ml}$ bovine serum albumin (BSA) for $60 \mathrm{~min}$ at $4^{\circ} \mathrm{C}$ with rocking and washed with PBS-PI three times. The beads were mixed with the cytosolic protein fractions and incubated for $16 \mathrm{~h}$ at $4^{\circ} \mathrm{C}$ with end-on-end mixing. Unbound proteins were removed by centrifuging the F-gel at $1000 \times g$ for $5 \mathrm{~min}$ and removing the supernatant. The F-gel was washed ten times with PBS-PI containing 0.1\% Triton-X100 before eluting bound proteins into sodium dodecyl sulfate (SDS)sample buffer (1M Tris pH 8.0, 20\% SDS, 0.5 M EDTA $\mathrm{pH} 8,10 \%$ glycerol, $200 \mathrm{mM}$ dithiothreitol). Bound proteins were resolved by SDS-PAGE and transferred to polyvinylidene difluoride membranes (Bio-Rad). Western blots were probed with antibodies to SseC (a gift from Dr. Michael Hensel), the FLAG epitope (Sigma), or the $\mathrm{His}_{6}$ tag (Qiagen). For reciprocal co-immunoprecipitations, a strain containing a plasmid encoding $s s c A-H I S_{6}$ and a second compatible plasmid encoding sseC-FLAG was used. SscA-His ${ }_{6}$ was induced with arabinose and SseC-FLAG was induced with IPTG as above. In this experiment, the anti-FLAG gel was used for immunoprecipitations and anti-His antibody used in immunoblotting as described above.

\section{Protein secretion assasy}

Wild type $S$. Typhimurium and $\triangle s s c A$ strains were grown overnight in LB and sub-cultured 1:50 into LPM and grown to $\mathrm{OD}_{600}$ of 0.6 . Cultures were then centrifuged for $2 \mathrm{~min}$ at $10,000 \times g$ and the supernatant was 
Table 1 Strains, plasmids and oligonucleotides used in this study

\begin{tabular}{|c|c|c|}
\hline Strains, plasmids and primers & Description & Reference or source \\
\hline \multicolumn{3}{|l|}{ Strains } \\
\hline SL1344 & Salmonella enterica serovar Typhimurium wild type & Our collection \\
\hline$\triangle s s c A$ & SL1344, deletion of SsCA & This study \\
\hline$\Delta s s e C$ & SL1344, deletion of sseC & This study \\
\hline SL1344 RES & SL1344, ushA::Cm; Cm $(10 \mu \mathrm{g} / \mathrm{ml})$ & [29] \\
\hline \multicolumn{3}{|l|}{ Plasmids } \\
\hline pKD4 & oriRy, $\operatorname{Kan}^{R}$ cassette flanked by FRT sites & [26] \\
\hline pKD46 & RepA1019(Ts), $\lambda, \gamma, \beta$ and exo expressed from ParaBAD, Amp ${ }^{R}$ & [26] \\
\hline pFLAG-CTC & Cytoplasmic expression of C-terminal FLAG fusion protein under control of the Ptac, Amp ${ }^{R}$ & Sigma \\
\hline psscA-FLAG & pFLAG-CTC, expresses in-frame fusion of SscA-FLAG & This study \\
\hline psseC-FLAG & pFLAG-CTC, expresses in-frame fusion of SseC-FLAG & This study \\
\hline pACYC184 & General cloning, $\mathrm{Tet}^{\mathrm{R}}, \mathrm{Cm}^{\mathrm{R}}$ & Our collection \\
\hline psscA-6His & pACYC; expresses in-frame fusion of SscA-6His & This study \\
\hline pBAD24 & Arabinose-inducible gene expression, pBR322ori, $\mathrm{P}_{\mathrm{BAD}}, r r n B T_{1,2}, \operatorname{araC}, \mathrm{Amp}^{\mathrm{R}}$ & [27] \\
\hline psscA & sseA-ssCA with endogenous sseA promoter in pWSK29, pSC101ori, Amp ${ }^{R}$ & This study \\
\hline \multicolumn{3}{|l|}{ Primers } \\
\hline sseC lambda-F & cgaattcacagtaatagcgacagcgccgcaggagtaaccgccttaacacagtgtaggctggagctgcttcg & \\
\hline sseC lambda-R & gcgatagccagctattctcgcctgaacgctactatagtgatcaatggtatcatatgaatatcctcctta & \\
\hline sscA lambda-F & gacccgaccctacaacaggcacatgacacgatgcggtttttccggcgtgggtgtaggctggagctgcttcg & \\
\hline sscA lambda-R & gtcagaaagttgctgtaacatcttttctgcacgctgtcggagaatttgatcatatgaatatcctcctta & \\
\hline$S S C A-F L A G-F$ & gcttaaagcttatgaaaaaagacccgaccct & \\
\hline$S S C A-F L A G-R$ & tatctgtcgacgctcctgtcagaaagttgct & \\
\hline sseC-FLAG-F & ggtcacatatgatgaatcgaattcacagtaa & \\
\hline sseC-FLAG-R & ggtcagtcgacagcgcgatagccagctattc & \\
\hline SSCA-HIS-F & gtcaggctagcaggaggatgcatcaccatcaccatcacatgaaaaaagacccgaccc & \\
\hline$S S C A-H I S-R$ & gtcagaagcttttagctcctgtcagaaagttg & \\
\hline SSCA-F & acgcgtcgacacaggatccgcagcaatatc & \\
\hline$S S C A-R$ & gctctagacccctaaatatgcaggctca & \\
\hline
\end{tabular}

filtered through a $0.2 \mu \mathrm{m}$ filter (Pall Scientific) and precipitated with $10 \%$ trichloroacetic acid (TCA). Precipitated secreted proteins were centrifuged at $16,000 \times g$ at $4^{\circ} \mathrm{C}$ for $30 \mathrm{~min}$ and the pellets were washed with acetone and dissolved in SDS-sample buffer. The whole cell lysate fraction was made by dissolving the bacterial pellet in SDS-sample buffer. To ensure equal protein loading in SDS-PAGE, the volume of SDS sample buffer was adjusted according to the optical density of the original culture. Protein samples were analyzed by Western blot using antibodies to DnaK (Convance), SseC (a gift from Dr. Michael Hensel), SseB, SseD, and SseG (a gift from Dr. John Brumell).

\section{Macrophage replication assays}

RAW264.7 cells were grown in Dulbecco's Modified Eagle Medium (DMEM; Gibco) supplemented with 10\% fetal bovine serum (FBS; Invitrogen) at $37^{\circ} \mathrm{C}$ with $5 \%$
$\mathrm{CO}_{2}$. Cells were seeded $16 \mathrm{~h}$ prior to infection into 24well plates at a density of $2 \times 10^{5}$ cells per well. Overnight cultures of bacteria were washed with PBS, diluted in DMEM/10\% FBS, and used to infect macrophages at a multiplicity of infection (MOI) of 50 for $30 \mathrm{~min}$ at $37^{\circ} \mathrm{C}, 5 \% \mathrm{CO}_{2}$. Infected cells were washed three times with PBS and the media was replaced with DMEM/10\% $\mathrm{FBS} / 100 \mu \mathrm{g} / \mathrm{mL}$ gentamicin for $1.5 \mathrm{~h}$ to kill extracellular bacteria. Cells were then washed twice with PBS and incubated for $20 \mathrm{~h}$ in DMEM/10\% FBS with $10 \mu \mathrm{g} / \mathrm{mL}$ gentamicin. At $2 \mathrm{~h}$ and $20 \mathrm{~h}$ after infection, the cells were washed twice with PBS then lysed with $1 \%$ Triton $\mathrm{X}-100,0.1 \%$ SDS in PBS to release intracellular bacteria. Colony forming units (cfu) were determined by plating serially-diluted lysates onto LB agar plates containing appropriate antibiotics. Experiments were performed twice independently using 3 technical replicates per assay. 


\section{Mouse infections}

Competitive infections were performed in female C57BL/6 mice (Charles River) by oral inoculation of a $0.1 \mathrm{ml} \mathrm{mix-}$ ture containing equal numbers $\left(1 \times 10^{8} \mathrm{cfu}\right)$ of a chloramphenicol resistant wild type strain $(u s h A:: \mathrm{Cm})$ and mutant strains as described previously [5]. The marked wild type strain was previously shown to be phenotypically neutral [30]. Three days after infection, the spleen, liver and cecum was removed, homogenized in ice-cold PBS (Mixer Mill, Retsch) and serially diluted in PBS. The competitive index $(\mathrm{CI})$ was determined by plating dilutions of the homogenized tissue lysates on agar plates containing streptomycin and incubating overnight at $37^{\circ} \mathrm{C}$ to recover both wild type and mutant bacteria. Colonies were then replica-stamped onto separate plates containing streptomycin and chloramphenicol to enumerate wild type and mutant bacteria. The CI was calculated as (cfu mutant/cfu wild type) $)_{\text {output }} /(\text { cfu mutant/cfu wild type })_{\text {input }}$. Mouse experiments were performed twice using groups of 5 mice for each experiment. Statistical analysis was performed using a Student $t$ test.

\section{Conclusion}

In summary, we have verified that SscA is the chaperone for the SseC translocon component in the T3SS encoded by SPI-2. This work completes the characterization of the known chaperone complement within SPI-2. In future work, it will be useful to investigate whether this particular chaperone-cargo pair has any additional regulatory function on gene expression within SPI-2.

\section{Abbreviations \\ SPI: Salmonella pathogenicity Island; T3SS: Type 3 secretion system; S. Typhimurium: Salmonella enterica serovar Typhimurium; TPR: Tetratricopeptide repeat; Cl: Competitive index.}

\section{Competing interests}

The authors indicate that there are no competing interests.

\section{Author's contributions}

Conducted experiments and analyzed data: CAC, DTM, SEA. Wrote manuscript: CAC, DTM, BKC. Edited manuscript and provided essential discussion: CAC, DTM, SEA, AVCP, BKC. All authors read and approved the final manuscript.

\section{Acknowledgements}

This work was supported by the Canadian Institutes of Health Research (CIHR; MOP-82704), the Natural Sciences and Engineering Research Council (NSERC) and the Canada Research Chairs program. CAC is the recipient of an NSERC postgraduate scholarship; DTM and SEA are each supported by a Canada Graduate Scholarship from the CIHR. BKC is the Canada Research Chair in Infectious Disease Pathogenesis.

\section{Author details}

${ }^{1}$ Michael G. DeGroote Institute for Infectious Disease Research, Hamilton, Ontario L8N 3Z5, Canada. 'Department of Biochemistry and Biomedical Sciences, McMaster University, Health Sciences Centre Room 4H17, Hamilton, Ontario L8S 4K1, Canada. ${ }^{3}$ Department of Molecular and Cellular Biology, University of Guelph, 488 Gordon Street, Science Complex, Room 4202, Guelph, Ontario N1G 2W1, Canada.
Received: 4 June 2013 Accepted: 1 October 2013

Published: 4 October 2013

\section{References}

1. Groisman EA, Ochman $\mathrm{H}$ : Cognate gene clusters govern invasion of host epithelial cells by Salmonella typhimurium and Shigella flexneri. EMBO J 1993, 12(10):3779-3787.

2. Shea JE, Hensel M, Gleeson C, Holden DW: Identification of a virulence locus encoding a second type III secretion system in Salmonella typhimurium. Proc Natl Acad Sci USA 1996, 93(6):2593-2597.

3. Ochman H, Soncini FC, Solomon F, Groisman EA: Identification of a pathogenicity island required for Salmonella survival in host cells. Proc Natl Acad Sci USA 1996, 93(15):7800-7804.

4. Cornelis GR: The type III secretion injectisome. Nat Rev Microbiol 2006, 4(11):811-825.

5. Cooper CA, Zhang K, Andres SN, Fang Y, Kaniuk NA, Hannemann M, Brumell JH, Foster LJ, Junop MS, Coombes BK: Structural and biochemical characterization of SrcA, a multi-cargo type III secretion chaperone in Salmonella required for pathogenic association with a host. PLOS pathogens 2010, 6(2):e1000751.

6. Luo Y, Bertero MG, Frey EA, Pfuetzner RA, Wenk MR, Creagh L, Marcus SL, Lim D, Sicheri F, Kay C, et al: Structural and biochemical characterization of the type III secretion chaperones CesT and SigE. Nat Struct Biol 2001, 8(12):1031-1036.

7. Stebbins CE, Galan JE: Maintenance of an unfolded polypeptide by a cognate chaperone in bacterial type III secretion. Nature 2001, 414(6859):77-81.

8. Buttner CR, Sorg I, Cornelis GR, Heinz DW, Niemann HH: Structure of the Yersinia enterocolitica type III secretion translocator chaperone SycD. J Mol Biol 2008, 375(4):997-1012.

9. Yip CK, Finlay BB, Strynadka NC: Structural characterization of a type III secretion system filament protein in complex with its chaperone. Nat Struct Mol Biol 2005, 12(1):75-81.

10. Parsot $C$, Hamiaux $C$, Page AL: The various and varying roles of specific chaperones in type III secretion systems. Curr Opin Microbiol 2003, 6(1):7-14.

11. Bennett JC, Thomas J, Fraser GM, Hughes C: Substrate complexes and domain organization of the Salmonella flagellar export chaperones FlgN and FliT. Mol Microbiol 2001, 39(3):781-791.

12. Francis MS, Lloyd SA, Wolf-Watz H: The type III secretion chaperone LcrH co-operates with YopD to establish a negative, regulatory loop for control of Yop synthesis in Yersinia pseudotuberculosis. Mol Microbiol 2001, 42(4):1075-1093.

13. Dai S, Zhou D: Secretion and function of Salmonella SPI-2 effector SseF require its chaperone, SscB. J Bacteriol 2004, 186(15):5078-5086.

14. Coombes BK, Brown NF, Kujat-Choy S, Vallance BA, Finlay BB: SseA is required for translocation of Salmonella pathogenicity island-2 effectors into host cells. Microbes Infect 2003, 5(7):561-570.

15. Ruiz-Albert J, Mundy R, Yu XJ, Beuzon CR, Holden DW: SseA is a chaperone for the SseB and SseD translocon components of the Salmonella pathogenicity-island-2-encoded type III secretion system. Microbiology 2003, 149(Pt 5):1103-1111.

16. Zurawski DV, Stein MA: SseA acts as the chaperone for the SseB component of the Salmonella Pathogenicity Island 2 translocon. Mol Microbiol 2003, 47(5):1341-1351.

17. Hensel M, Shea JE, Waterman SR, Mundy R, Nikolaus T, Banks G, Vazquez Torres A, Gleeson C, Fang FC, Holden DW: Genes encoding putative effector proteins of the type III secretion system of Salmonella pathogenicity island 2 are required for bacterial virulence and proliferation in macrophages. Mol Microbiol 1998, 30(1):163-174.

18. Altschul SF, Madden TL, Schaffer AA, Zhang J, Zhang Z, Miller W, Lipman DJ: Gapped BLAST and PSI-BLAST: a new generation of protein database search programs. Nucleic Acids Res 1997, 25(17):3389-3402.

19. Edqvist PJ, Broms JE, Betts HJ, Forsberg A, Pallen MJ, Francis MS: Tetratricopeptide repeats in the type III secretion chaperone, LcrH: their role in substrate binding and secretion. Mol Microbiol 2006, 59(1):31-44

20. Schreiner M, Niemann HH: Crystal structure of the Yersinia enterocolitica type III secretion chaperone SycD in complex with a peptide of the minor translocator YopD. BMC Struct Biol 2012, 12:13. 
21. Pollastri G, Przybylski D, Rost B, Baldi P: Improving the prediction of protein secondary structure in three and eight classes using recurrent neural networks and profiles. Proteins 2002, 47(2):228-235.

22. Lunelli M, Lokareddy RK, Zychlinsky A, Kolbe M: IpaB-IpgC interaction defines binding motif for type III secretion translocator. Proc Natl Acad SCi USA 2009, 106(24):9661-9666.

23. Nikolaus T, Deiwick J, Rappl C, Freeman JA, Schroder W, Miller SI, Hensel M: SseBCD proteins are secreted by the type III secretion system of Salmonella pathogenicity island 2 and function as a translocon. J Bacteriol 2001, 183(20):6036-6045.

24. Sory MP, Cornelis GR: Translocation of a hybrid YopE-adenylate cyclase from Yersinia enterocolitica into HeLa cells. Mol Microbiol 1994, 14(3):583-594.

25. Edqvist PJ, Aili M, Liu J, Francis MS: Minimal YopB and YopD translocator secretion by Yersinia is sufficient for Yop-effector delivery into target cells. Microbes Infect 2007, 9(2):224-233.

26. Datsenko KA, Wanner BL: One-step inactivation of chromosomal genes in Escherichia coli K-12 using PCR products. Proc Natl Acad Sci USA 2000, 97(12):6640-6645.

27. Guzman LM, Belin D, Carson MJ, Beckwith J: Tight regulation, modulation, and high-level expression by vectors containing the arabinose PBAD promoter. J Bacteriol 1995, 177(14):4121-4130.

28. Coombes BK, Wickham ME, Brown NF, Lemire S, Bossi L, Hsiao WW, Brinkman FS, Finlay BB: Genetic and molecular analysis of GogB, a phage-encoded type III-secreted substrate in Salmonella enterica serovar typhimurium with autonomous expression from its associated phage. J Mol Biol 2005, 348(4):817-830.

29. Brown NF, Vallance BA, Coombes BK, Valdez $Y$, Coburn BA, Finlay BB: Salmonella pathogenicity island 2 is expressed prior to penetrating the intestine. PLoS pathogens 2005, 1(3):e32.

30. Coombes BK, Wickham ME, Lowden MJ, Brown NF, Finlay BB: Negative regulation of Salmonella pathogenicity island 2 is required for contextual control of virulence during typhoid. Proc Natl Acad Sci USA 2005, 102(48):17460-17465.

doi:10.1186/1471-2180-13-221

Cite this article as: Cooper et al.: The $\mathrm{SseC}$ translocon component in

Salmonella enterica serovar Typhimurium is chaperoned by SscA. BMC Microbiology 2013 13:221

\section{Submit your next manuscript to BioMed Central and take full advantage of:}

- Convenient online submission

- Thorough peer review

- No space constraints or color figure charges

- Immediate publication on acceptance

- Inclusion in PubMed, CAS, Scopus and Google Scholar

- Research which is freely available for redistribution 